\title{
Exploration of Biostatistics Research in the New Century
}

\author{
Xiao Xiaonan \\ Xiamen University Tan Kah Kee College \\ Zhangzhou, Fujian, China \\ xiaoxn@xujc.com
}

\begin{abstract}
This paper discusses the main objects and scope of Biostatistics research in the new century, as well as the characteristics and methods of Biostatistical research in the new century. The results of the discussion show that the continuous deepening of the research on Biostatistics theory and methods will further open up the ideas and ways for people to enter new fields, and will explore the mysteries of life phenomena and raise human understanding of the world of life to another new level.
\end{abstract}

Keywords-Biostatistics; Life phenomenon; Mathematical model; Optimal decision

\section{RESEARCH TASKS AND SCOPE OF BIOSTATISTICS IN THE NEW CENTURY}

Biostatistics is a new subject that uses statistical theory and methods to study the quantitative relationship in biological sciences.

It is an extremely important sub-discipline in the Grand Statistics Department. The main research object of Biostatistics is the law of various quantitative changes in biological (life) science. Its main research task is to obtain statistical information through theoretical and numerical analysis, and provide the necessary scientific basis for the quantitative study of biological phenomena. It profoundly reveals the quantitative regularity hidden inside the biological (life) phenomenon. And then it explains and predicts the occurrence and development of certain biological (life) phenomena, the prediction and prevention of environmental pollution, the management and development of natural resources, etc. Thus it provides a reliable guarantee for people to make optimal decisions, [1-3].

The content of Biostatistics is very rich and covers a wide range. It includes biological sampling estimation, biological experimental design, biological variance analysis, biological regression analysis, biological multivariate statistics, biological life estimation, biological time series process, biological data filtering and biological quality assessment. In the past 50 years, the deepening of the research on the theory and method of Biostatistics will further open up the ideas and ways for people to enter new fields, and promote the continuous development of Biostatistics research [4-5].

\section{RESEARCH CHARACTERISTICS AND METHODS OF BIOSTATISTICS IN THE NEW CENTURY}

Since the twentieth century, with the mutual penetration of statistics and biology, the application of statistics has changed from non-life to life, which has led to the emerging edge discipline of Biostatistics. So it can be seen that the characteristics of life phenomena determine the characteristics of Biostatistics.

Since life activities occur in a large number of repetitions and periodic cycles accompanied by many random factors, the application of probability and statistics methods to the randomness of life phenomena has become the first feature of Biostatistics research.

Everything in the world is interrelated and mutually restrictive, and life phenomena are particularly prominent. Those one-sided, isolated, static, and mechanical research methods can no longer meet the needs of the development of biological sciences. Only comprehensive, athletic, and interrelated comprehensive statistical methods can meet the needs of biological research. This is the second feature of Biostatistical research

It is necessary to study not only the continuity of biological (life) phenomena, but also the corresponding statistical methods to study the discontinuity of the structure of living matter and the way of life activities. This is the third feature of Biostatistical research

In short, the phenomenon of life is a high-level form of complex and variable material movement that is both interconnected and mutually constrained. The research method is fundamentally different from the non-life science research. We must never copy the research method of non-life science. Rather, it is necessary to find a set of statistical methods for quantitatively studying the quantitative law of random phenomena in biological sciences to reveal the inherent regularity of random variables in biological sciences. Biostatistics has become a relatively complete and mature branch of the major statistical discipline because it adapts to the randomness of life phenomena[6-8]. 


\section{PROGRESS AND CURRENT SITUATION OF BIOSTATISTICS IN THE NEW CENTURY}

In the past 50 years, in addition to the application of biostatistical testing methods and improved research, with the rapid development of life sciences, survival analysis and life table research have become an important part of current Biostatistical research. It is widely used in the fields of demographics, medical follow-up, ecology, biological control, etc.

Modern Biostatistics research continues to develop in depth. Multi-state survival analysis has become a new topic combining biological stochastic processes with mathematical statistics. Multivariate biostatistics has been widely used in biomedicine. The combination of biostatistics and computer has become an important symbol of biomedical modernization. The recent etiology-associated model, disease discriminant analysis and prediction system, malignant ovarian tumor diagnosis model, internal medicine diagnosis system, etc., is the application of multi-index, multivariate biostatistical analysis in clinical medical research. In order to improve medical diagnosis to an expert level and minimize the rate of misdiagnosis, the following modes can be used to achieve: Expert treatment experience $\rightarrow$ mathematics (statistical information, knowledge expression) $\rightarrow$ computer learning $\rightarrow$ feedback correction $\rightarrow$ expert consultation system $\rightarrow$ computer consultation. This modern computer inquiry mode, which integrates biology, statistics and computer science, will certainly promote breakthroughs in modern biomedical science and technology [9-10].

\section{EXPECTATION}

The development of Biostatistics has promoted the development of biological sciences, which in turn has promoted the development of big statistics itself. It is pushing modern statistics to a new level. The main hotspots of current Biostatistical research are the researches on ecological life science issues, such as the development and utilization of biological resources, ecological management and protection, population growth and control, optimal management of agriculture, forestry and animal husbandry, analysis and treatment of environmental pollution, epidemics Epidemic patterns and their prediction, prevention, control, etc. They are the most interesting research topics in recent years [11-12].

The main task of Biostatistics is how to establish an optimized mathematical model that is in line with the reality of biological ecology. Due to the complexity of biological science, its research is often difficult. The required statistical knowledge and methods are also more comprehensive. Therefore, biostatisticians must pay attention to the renewal of existing statistical methods and the further understanding of life sciences.

With the continuous deepening of China's reform and opening up and the promotion of international trends, the research on life sciences has greatly improved in China. People are looking forward to taking off. Some scientists predict that the 21 st century will be the century of life science. The deeper and more thorough the life phenomenon is revealed, the more statistical knowledge you need to use. Biological sciences, which are advancing rapidly, will undoubtedly reveal the essence of life phenomena more profoundly under the impetus of the theories and methods of big statistics. Biostatistics will become an indispensable tool for the study of bioscience, and will explore the mysteries of life phenomena, as well as to raise human understanding of the world of life to a new level. The times are moving forward. Science is progressing. Let us give full play to wisdom of the Chinese nation and rely on science to promote our Chinese culture. Let us make a new record and new contribution to the development of the major statistics and life sciences.

\section{REFERENCES}

[1] S Yang, M Santillana, S C Kou. Accurate estimation of influenza epidemics using Google search data via ARGO[J]. Proceedings of the National Academy of Sciences of United States of America. 2015(112):14473-14478.

[2] C Kao. Network data envelopment analysis: A review[J].European Journal of Operational Research, 2014(2):1-16

[3] M Santillana,et al. What can digital diseasedetection learn from (an external revision to) Google Flu Trends[J]. American Journal of Preventive Medicine,2014(47):341-347.

[4] J Ginsberg, M H Mohebbi, R S Patel. Detecting influenza epidemics using search engine query data [J]. Nature, 2009(457):1012-1014.

[5] M Santillana, et al. Using clinicians' search query data to monitor influenza epidemics[J].Clinical Infectious Diseases,2014(59):1446-1450.

[6] M J Paul, M Dredze, D Broniatowski. Twitter improves influenza forecasting [J]. PLOS Currents Outbreaks. 2014 Oct 28. Edition 1.

[7] L Simar,A Vanhems, Probabilistic characterization of directional distances and their robust versions[J].Journal of Econometrics,2012(2):342-354.

[8] J McDounald. Using least squares and tobit in second stage DEA efficiency analysis[J]. European Journal of Operational Research,2009(2):792-798.

[9] BU Park, L simar, V Zelenyuk. Local likelihood estimation of trumcated regression and its partial derivative: theory and application[J]. Journal of Econometrics, 2008(1):185-198.

[10] S Reinhard, CAK Lovell, G Thijssen. Environmental efficiency with multiple environmentally detrimental variables estimated with SA and DEA[J]. European Journal of Operational Research,2000(2):287-303.

[11] L Badin, C Daraio, L Simar. Optimal bandwidth selection for conditional efficiency measures: a data-driven approach $[\mathrm{J}]$. European Journal of Operational Research, 2010(2):633-640.

[12] D Lazer, R Kennedy, G King, A Vespignani. The parable of Google Flu: Traps in big data analisis[J]. Science, 2014(343):1203-1205. 